\title{
Metformin use and health care utilization in patients with coexisting chronic obstructive pulmonary disease and diabetes mellitus
}

This article was published in the following Dove Press journal: International Journal of COPD

\author{
Raju Bishwakarma' \\ Wei Zhang' \\ Yu-Li Lin² \\ Yong-Fang Kuo $\mathrm{K}^{2,3}$ \\ Victor J Cardenas' \\ Gulshan Sharma ${ }^{1,3}$ \\ 'Division of Pulmonary, Critical Care \\ and Sleep Medicine, Department \\ of Internal Medicine, ${ }^{2}$ Office of \\ Biostatistics, ${ }^{3}$ Sealy Center on Aging, \\ University of Texas Medical Branch, \\ Galveston, TX, USA
}

Background: Chronic obstructive pulmonary disease (COPD) is associated with persistent systemic inflammation. Anti-inflammatory therapies have been shown to decrease acute exacerbations of COPD. The antidiabetic medication metformin decreases oxidative stress and inflammation and may benefit patients with COPD. We aimed at investigating the effect of metformin on health care utilizations in patients with coexisting COPD and diabetes mellitus (DM).

Methods: We studied 5\% Medicare beneficiaries with coexisting COPD and DM prescribed metformin or other antidiabetics during the period 2007-2010. The primary outcome was COPD-specific emergency room (ER) visits and hospitalizations; the secondary outcome was all-cause ER visits and hospitalizations over the 2-year follow-up after the index antidiabetic prescription. The effects of metformin were examined by COPD complexity and compared with the effects of other antidiabetic medications.

Results: Among 11,260 patients, 3,193 were metformin users and 8,067 were nonusers. Metformin users were younger, were less sick, were less likely to be on oxygen, and had fewer hospitalizations in the prior year compared with the nonusers. Over a 2-year period, metformin users had lower COPD-specific and all-cause ER visits and hospitalizations ( $7.11 \%$ vs $9.61 \%$, $p<0.0001$; and $61.63 \%$ vs $71.27 \%, p<0.0001$, respectively). In a stratified multivariable analysis, the odds of COPD-specific ER visits and hospitalizations were lower in patients with low-complexity COPD (adjusted odds ratio $=0.66,95 \%$ confidence interval $=0.52-0.85$ ). However, patients with all COPD complexities get benefits of metformin on all-cause ER visits and hospitalizations.

Conclusion: The use of metformin in patients with coexisting COPD and DM was associated with fewer COPD-specific ER visits and hospitalizations, especially in low-complexity COPD.

Keywords: COPD, diabetes, metformin, ER visits, hospitalization, Medicare

\section{Introduction}

The prevalence of chronic obstructive pulmonary disease (COPD) increases with aging, and diabetes mellitus (DM) is a common comorbidity in patients with COPD. ${ }^{1-3}$ Inflammation is a common feature in all three conditions: COPD, DM, and aging. ${ }^{1,3,4}$ Various anti-inflammatory therapies such as phosphodiesterase-4 inhibitor, N-acetylcysteine, carbocisteine, and macrolide have been shown to reduce airway inflammation and prevent acute exacerbations of COPD..$^{5-8}$

Metformin, a biguanide, is a first-line antidiabetic drug with diverse pleotropic properties. Metformin activates both adenosine monophosphate-activated protein kinase (AMPK)-dependent and AMPK-independent pathways, resulting in reduction in oxidative
Correspondence: Raju Bishwakarma Valley Health, Winchester Medical Center, 1840 Amherst Street, Winchester, VA 2260I, USA

Tel +I 5405367087

Fax +I 5405366625

Email rajucentury@gmail.com
International Journal of COPD 2018:13 793-800

793

(c) (1) (5) 2018 Bishwakarma et al. This work is published and licensed by Dove Medical Press Limited. The full terms of this license are available at https://www.dovepress.com/terms.php (c)
hereby accept the Terms. Non-commercial uses of the work are permitted without any further permission from Dove Medical Press Limited, provided the work is properly attributed. For permission for commercial use of this work, please see paragraphs 4.2 and 5 of our Terms (https://www.dovepress.com/terms.php). 
stress and chronic inflammation. ${ }^{9,10}$ Animal and clinical studies have suggested other beneficial effects of metformin besides diabetes control. Metformin decreases inflammatory cytokines, such as tumor necrosis factor- $\alpha$, interleukin (IL)-6, and IL-1, and the inflammatory response of macrophages and induces the production of anti-inflammatory cytokines such as IL-4 and IL-10. ${ }^{11,12}$ In the animal model, metformin has been shown to decrease eosinophilic inflammation, airway remodeling, and peribronchial and parenchymal fibrosis. ${ }^{13-15}$ In clinical studies, metformin use in patients with COPD and $\mathrm{DM}$ is associated with improvement in respiratory symptoms, St George Respiratory Questionnaire (SGRQ) and transitional dyspnea index scores, respiratory muscle function, ${ }^{16}$ and overall survival among long-term users. ${ }^{17}$

It is plausible that metformin use in patients with COPD decreases both local and systemic inflammation and oxidative damage. Our hypothesis was that patients with coexisting COPD and DM on metformin have lower health care utilization as measured by percentage of patients with an emergency room (ER) visits and hospitalizations during a 2-year period. Our primary outcome of interest was COPD-specific ER visits and hospitalizations, and the secondary outcome was all-cause ER visits and hospitalizations.

\section{Methods}

\section{Data source}

This is a retrospective study using 5\% Medicare beneficiaries' data from the year 2006 to 2012 . Over $98 \%$ of adults in the USA aged $\geq 65$ years were enrolled in Medicare, which serves $>45$ million beneficiaries. The Centers for Medicare and Medicaid Services selected a random sample of 5\% Medicare beneficiaries based on the eighth and ninth digits $(05,20,45,70$, and 95) of their health insurance claim number. This population is the standard available for research purposes and has been shown to be representative of the whole cohort. Medicare Beneficiary Summary Files, Medicare Provider Analysis and Review (MedPAR) Files, Outpatient Standard Analytical Files (OutSAFs), Medicare Carrier Files, Durable Medical Equipment (DME) Files, and Prescription Drug Event (PDE) records ${ }^{18}$ were used for the study. We also used the Chronic Condition Data Warehouse (CCW) to identify patients with coexisting COPD and DM. ${ }^{19}$ The CCW data were linked by a unique, unidentifiable beneficiary key, which allows researchers to analyze information across the continuum of care. The $27 \mathrm{CCW}$ chronic condition categories include COPD and DM, available from 1999. ${ }^{19}$ The Research Data Assistance Center provided deidentified data for the research purpose and, due to the nature of the study, patient consent was not required. The study was approved by the University of Texas Medical Branch Institutional Review Board (Project 09-054).

\section{Study cohort}

Figure 1 shows the establishment of the study cohort. The study cohort consisted of patients with both COPD and DM, who received antidiabetic treatment between 2007 and 2010. First, we selected beneficiaries who had both COPD and DM between 2007 and 2010 by using CCW chronic condition flags and identified those who had any antidiabetic prescription in the same period. We included those who had their first antidiabetic prescription either in the same year when both COPD and DM were diagnosed or in the year after. The date of the first antidiabetic prescription between 2007 and 2010 was used as the index date.

We then selected those who had complete Medicare Parts A, B, and D enrollment in the year prior to and 2 years after the index date. In order to ensure that the effects observed would be associated with a particular antidiabetic, we excluded those who switched between metformin and non-metformin or those who did not renew the antidiabetic prescription. Lastly, we excluded those who were younger than 66 years, who had end-stage renal disease (ESRD)/ disability as the original reason for enrollment, who had ESRD in the previous year and in the following 2 years, who had hemodialysis in the prior year, or who were in a nursing facility in the prior year. Hemodialysis was identified by Current Procedural Terminology (CPT) codes 90935, 90937, 90940, and 99512 from OutSAFs and Medicare Carrier Files, and nursing facility services were identified by using CPT codes 99301-99313, 99315, 99316, and 99318 from Medicare Carrier Files. Among these 11,260 patients, 3,193 were metformin users; the rest 8,067 used other antidiabetics, including insulin, sulfonylurea, acarbose, exenatide, glucagon, miglitol, nateglinide, pioglitazone, pramlintide, repaglinide, rosiglitazone, saxagliptin, and sitagliptin.

\section{Measures}

The patients were categorized by age, gender, race/ethnicity, and socioeconomic status. We used the Medicare-Medicaid dual eligibility as a proxy of low socioeconomic status. Total hospitalizations in the prior year were identified from MedPAR Files. Oxygen use in the prior year was identified by Healthcare Common Procedure Coding System codes E1390, E1391, and E1392 from DME Files. Spirometry was identified through CPT codes (94010, 94014, 94015, 94016, 94060, 94070, and 94620). Medication for COPD 


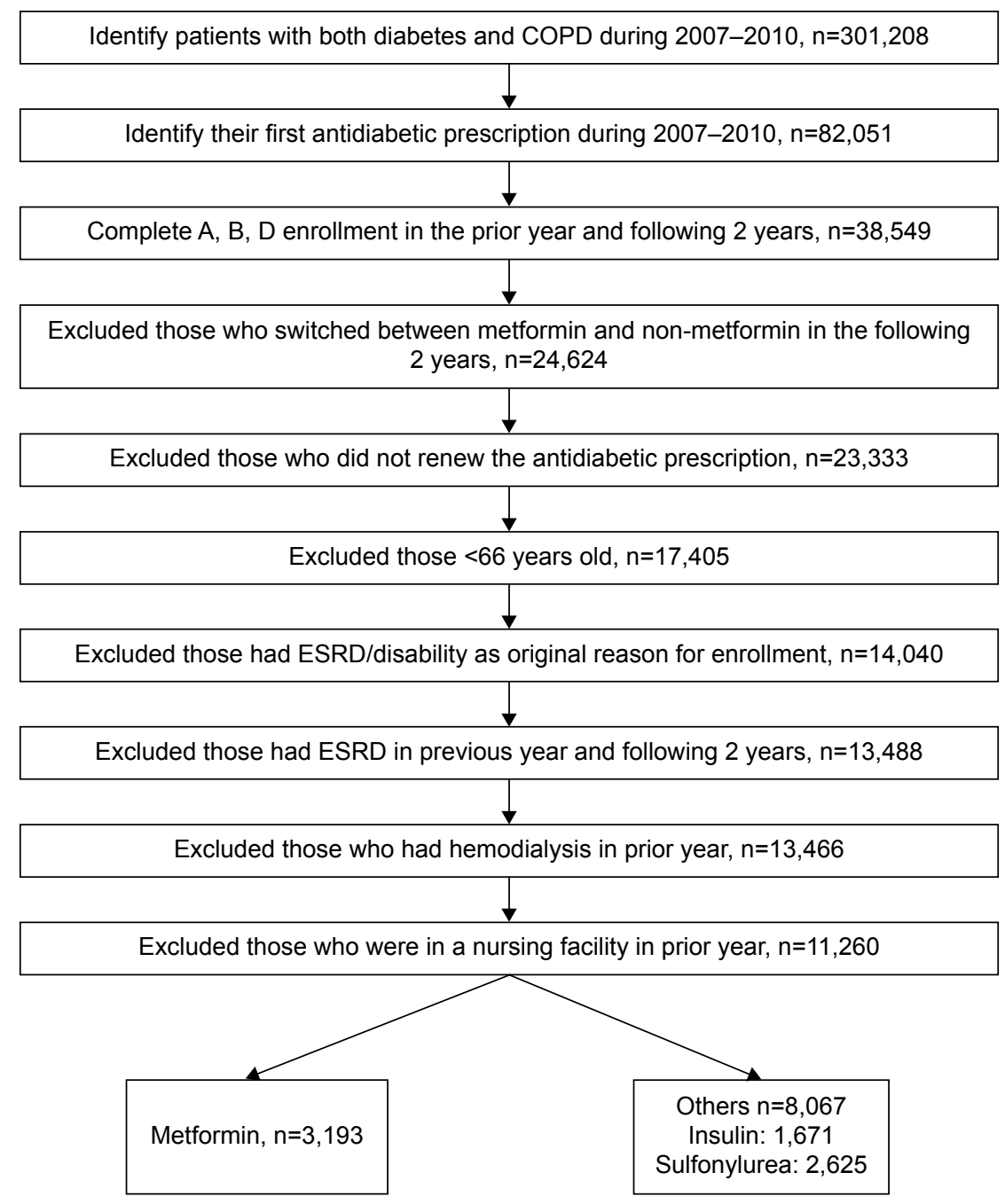

Figure I Establishment of the cohort.

Abbreviations: COPD, chronic obstructive pulmonary disease; ESRD, end-stage renal disease.

in the follow-up period, including long-acting $\beta$-agonists (LABAs), long-acting muscarinic antagonists (LAMAs), inhaled corticosteroids (ICS), ICS with LABAs, shortacting $\beta$-agonists, and short-acting muscarinic antagonist, was identified from PDE records.

The following conditions in the prior year were identified by either one inpatient (MedPAR) claim or two outpatient/physician (Carrier) claims at least 30 days apart with the following International Classification of Diseases, Ninth Revision, Clinical Modification (ICD-9-CM) codes: gastroesophageal reflux disease (GERD; 530.x-535.x); lung cancer (162.x); anxiety/depression (296.2, 296.3, 296.5, 300.0, 300.4, 309.x, and 311.x); sarcopenia (728.2); osteoporosis (733.0); cardiovascular disease (CVD; including ischemic heart disease; 410.x, 411.x, 412.x, 413.x, and 414.x), cerebrovascular disease (433.x, 434.x, 435.x, 436.x, 437.x, and 438.x), arrhythmia (427.x), heart failure (428.x), and artery disease (440.x, 441.x, 442.x, 443.2x, 443.9x, and 444.x).

To adjust for severity of COPD and DM, COPD severity was categorized as low, moderate, and high complexity as described by Mapel et al, ${ }^{20}$ and DM was categorized as uncontrolled DM and DM with complications, as published earlier. $^{21}$

\section{Study outcomes}

The primary outcome of this study was COPD-specific ER visits and hospitalizations in the 2 years following the index antidiabetic prescription. The secondary outcome was all-cause ER visits and hospitalizations. ER visit was identified by the provider-assigned revenue codes $0450-0459$ and 0981 from claims in OutSAFs. Hospitalizations were identified using MedPAR claims. COPD-specific diagnoses (ER visits and hospitalizations) were identified by claims 
with ICD-9-CM codes 491.21, 491.22, 491.8, 491.9, 492.8, 493.20, 493.21, 493.22, and 496.x as the primary diagnosis or claims with ICD-9-CM codes 518.81, 518.82, 518.84, and 799.1 as the primary diagnosis and 491.21, 491.22, 493.21, and 493.22 as one of the secondary diagnosis. All others were defined as all-cause ER visits and hospitalizations.

\section{Statistical analyses}

We calculated the proportion of patients with COPD-specific and all-cause ER visits and hospitalizations during the follow-up period for metformin user and nonuser groups. The distributions of patient characteristics were calculated, and the difference among groups was examined by $\chi^{2}$ tests or Fisher's exact test. The difference in COPD-specific and all-cause outcomes among groups was examined by $\chi^{2}$ tests. The effect of group characteristics (metformin users vs nonusers) on the likelihood of having any COPD-specific or all-cause ER visit or hospitalization was examined by logistic regression, adjusted for age, gender, race, dual eligibility, prior-year hospitalization, COPD complexity, diabetes complexity, comorbidities (GERD, anxiety and depression, CVD, sarcopenia, and osteoporosis), prior-year oxygen use, and COPD maintenance medications use. The effect of metformin was stratified by the severity of COPD (low complexity vs moderate/high complexity) on these outcomes. The secondary analysis was performed to study the effect of individual antidiabetic medications (categorized as metformin, sulfonylurea, insulin, and other) on the incidence of COPD-specific and all-cause ER visits and hospitalizations. The "other" group included those who were on one or more antidiabetics other than insulin, metformin, and sulfonylurea. All analyses were performed with SAS Version 9.4 (SAS Inc., Cary, NC, USA). All reported $p$-values were two-sided, and $p<0.05$ was considered statistically significant.

\section{Results}

Table 1 shows the baseline characteristics of the 11,260 Medicare patients with coexisting COPD and DM who had received antidiabetic medication from 2007 to 2010 and were followed up for 2 years. Of these, 3,193 patients were on metformin and 8,067 were on other antidiabetic medications.

Metformin users were younger, were less sick with fewer cardiovascular comorbidities, were less likely to be on oxygen, and had better controlled diabetes with fewer complications and fewer hospitalizations in the prior year compared with metformin nonusers. The two groups had no significant difference in sarcopenia, osteoporosis, anxiety and depression, and GERD. In addition, the two groups had no significant difference in receiving spirometry in the prior year and the following 2 years. Metformin nonusers had more complex COPD and were more likely to be on COPD maintenance medications such as LABA/LAMA and/or ICS.

Table 2 shows health care-related outcomes over a 2-year period in metformin users vs nonusers. COPD-specific and all-cause ER visits and hospitalizations were lower in metformin users $(7.11 \%$ vs $9.61 \%, p<0.0001$; and $61.63 \%$ vs $71.27 \%, p<0.0001$, respectively) compared with metformin nonusers. Outcomes varied by COPD complexity. Patients with low-complexity COPD had fewer COPD-specific ER visits and hospitalizations, whereas patients with low- and moderate-complexity COPD had fewer all-cause ER visits and hospitalizations.

We also examined the interaction effects of severity of COPD and types of antidiabetic users on all-cause and COPD-specific ER visits and hospitalizations. Although the interactions were not clinically significant, it is important to assess the effect of metformin stratified by the severity of COPD (low complexity vs moderate/high complexity) on these outcomes. Table 3 presents the odds of ER visits and hospitalizations based on the complexity of COPD after adjusting for baseline characteristics as shown in Table 1, except spirometry to avoid collinearity with COPD complexity. Only those with low-complexity COPD had significantly lower odds of COPD-specific ER visits and hospitalizations (adjusted odds ratio $[\mathrm{OR}]=0.66 ; 95 \%$ confidence interval $[\mathrm{CI}]=0.52-0.85)$. Metformin users had lower rates of allcause ER visits and hospitalizations irrespective of COPD complexity.

Table 4 shows the effect of each antidiabetic medication on COPD outcomes. Among 8,067 metformin nonusers, 1,671 used only insulin, 2,625 used only sulfonylureas, and 3,771 used other antidiabetics over a 2-year period. Among all the antidiabetics, metformin had the highest reduction in odds for COPD-specific ER visits and hospitalizations, especially in patients with low-complexity COPD.

\section{Discussion}

Our study results can be summarized as follows: patients with coexisting COPD and DM who used metformin had lower health care utilization as compared to nonusers. The most benefit is found in patients with low COPD complexity.

The findings of our study build upon prior studies of metformin use in patients with COPD. Sexton et al conducted an open-label observational study in 17 patients with COPD and diet-controlled diabetes or impaired glucose tolerance. Metformin was given daily to all participants for 6 months. 
Table I Baseline characteristics of patients with coexisting COPD and DM during 2007-20I0 by metformin use

\begin{tabular}{|c|c|c|c|}
\hline \multirow[t]{2}{*}{ Patient characteristics } & \multicolumn{2}{|l|}{ Metformin use } & \multirow[t]{2}{*}{$p$-value ${ }^{c}$} \\
\hline & $\mathrm{Yes}^{\mathrm{a}}(\mathrm{n}=3,193)$ & $\mathrm{No}^{\mathrm{b}}(\mathrm{n}=\mathbf{8 , 0 6 7})$ & \\
\hline Age (years) & & & $<0.0001$ \\
\hline $66-74$ & $\mathrm{I}, 50 \mathrm{I}(47.0 \mathrm{I})$ & $2,737(33.93)$ & \\
\hline $75-84$ & $\mathrm{I}, 400(43.85)$ & $3,958(49.06)$ & \\
\hline $85+$ & $292(9.15)$ & $\mathrm{I}, 372(17.0 \mathrm{I})$ & \\
\hline Gender & & & 0.16 \\
\hline Male & I,042 (32.63) & $2,522(31.26)$ & \\
\hline Female & $2,151(67.37)$ & $5,545(68.74)$ & \\
\hline Race/ethnicity & & & $<0.0001$ \\
\hline White & $2,513(78.70)$ & 5,775 (7I.59) & \\
\hline Non-white & $680(21.30)$ & $2,292(28.4 I)$ & \\
\hline Dual eligibility & I,268 (39.7I) & $4,408(54.64)$ & $<0.0001$ \\
\hline Hospitalizations, prior year & & & $<0.0001$ \\
\hline 0 & $2,474(77.48)$ & 5,509 (68.29) & \\
\hline I & $506(15.85)$ & I,592 (19.73) & \\
\hline $2+$ & $213(6.67)$ & $966(11.97)$ & \\
\hline COPD complexity & & & 0.036 \\
\hline Low & $2,354(73.72)$ & 5,757 (7I.36) & \\
\hline Moderate & $704(22.05)$ & I,958 (24.27) & \\
\hline High & I35 (4.23) & $352(4.36)$ & \\
\hline Complications of DM & $472(14.78)$ & $2,697(33.43)$ & $<0.000$ I \\
\hline Uncontrolled DM & $672(21.05)$ & $2,802(34.73)$ & $<0.0001$ \\
\hline Oxygen use, prior year & $371(11.62)$ & I, I80 (14.63) & $<0.0001$ \\
\hline Spirometry, prior year and following 2 years & $960(30.07)$ & $2,319(28.75)$ & 0.17 \\
\hline \multicolumn{4}{|l|}{ Comorbidities } \\
\hline GERD, prior year & $382(11.96)$ & $987(12.24)$ & 0.69 \\
\hline Anxiety/depression, prior year & $26 \mid(8.17)$ & $639(7.92)$ & 0.66 \\
\hline CVD, prior year & $\mathrm{I}, 335(4 \mathrm{I} .8 \mathrm{I})$ & $4,011(49.72)$ & $<0.0001$ \\
\hline Sarcopenia, prior year & $4(0.13)$ & $8(0.10)$ & 0.70 \\
\hline Osteoporosis, prior year & $149(4.67)$ & $384(4.76)$ & 0.83 \\
\hline COPD medication, 2-year follow-up & & & 0.0001 \\
\hline Long-acting medication only & $378(11.84)$ & $834(10.34)$ & \\
\hline Short-acting medication only & $427(13.37)$ & I,329 (16.47) & \\
\hline Both & $625(19.57)$ & $\mathrm{I}, 598(19.8 \mathrm{I})$ & \\
\hline None & $\mathrm{I}, 763(55.2 \mathrm{I})$ & $4,306(53.38)$ & \\
\hline
\end{tabular}

Notes: ${ }^{\mathrm{a}}$ Those who had coexisting COPD and DM and received prescription for metformin during 2007-2010. ${ }^{\mathrm{b}}$ Those who had coexisting COPD and DM and received antidiabetics prescription other than metformin, such as insulin, sulfonylurea, acarbose, exenatide, glucagon, miglitol, nateglinide, pioglitazone, pramlintide, repaglinide, rosiglitazone, saxagliptin, or sitagliptin during 2007-2010. ' $\chi 2$ test and Fisher's exact test for sarcopenia. Data presented as $\mathrm{n}$ (\%) unless otherwise indicated.

Abbreviations: COPD, chronic obstructive pulmonary disease; CVD, cardiovascular disease; DM, diabetes mellitus; GERD, gastroesophageal reflux disease.

In comparison with baseline, follow-up at 26 weeks showed improvement in transitional dyspnea score, SGRQ score, and improved respiratory muscle strength PImax. ${ }^{16}$ In a single-center retrospective study, Hitchings et al followed 130 patients with COPD and DM, finding that, overall, metformin users had better survival (5.2 years, 95\% $\mathrm{CI}=4.5-5.8$, vs 1.9 years, $95 \% \mathrm{CI}=1.1-2.6) .{ }^{17} \mathrm{Li}$ et al showed that metformin users had fewer asthma-related hospitalizations $(\mathrm{OR}=0.21,95 \% \mathrm{CI}=0.07-0.63)$ and asthma exacerbation $(\mathrm{OR}=0.39,95 \% \mathrm{CI}=0.19-0.79)$ as compared to nonusers. ${ }^{22}$

The biological plausibility of the beneficial effects of metformin in patients with COPD is likely related to its pleotropic properties. Metformin is shown to decrease airway inflammation, airway remodeling, peribronchial and parenchymal fibrosis, and pro-inflammatory cytokines and to induce the production of anti-inflammatory cytokines. ${ }^{11-15}$ Skeletal muscle dysfunction is common in elderly adults ${ }^{23}$ and in those with $\mathrm{COPD}^{3,24}$ and DM. ${ }^{25}$ Sarcopenia is associated with poor outcomes in COPD. ${ }^{24,26}$ Metformin enhances skeletal muscle function by improving mitochondrial biogenesis in muscle and activating AMPK. ${ }^{27,28}$

With worsening airflow limitation, the degree of inflammation worsens in patients with COPD, predisposing them to systemic comorbidities..$^{29,30}$ Indeed, cardiovascular and other comorbidities are higher in COPD patients with moderate and severe diseases as compared to those with mild disease, ${ }^{31-33}$ perhaps reflecting that the common inflammatory pathway 
Table 2 ER visits and hospitalizations over a 2-year follow-up period in patients with coexisting COPD and DM

\begin{tabular}{|c|c|c|c|}
\hline \multirow{2}{*}{$\begin{array}{l}\text { ER visits and } \\
\text { hospitalizations }\end{array}$} & \multicolumn{2}{|c|}{ Metformin users } & \multirow[t]{2}{*}{$p$-value } \\
\hline & Yes $^{a}$ n (\%) & $\mathrm{No}^{\mathrm{b}}$ n (\%) & \\
\hline COPD-specific (overall) & $227(7.11)$ & $775(9.61)$ & $<0.0001$ \\
\hline \multicolumn{4}{|l|}{ COPD complexity } \\
\hline Low & $97(4.12)$ & $350(6.08)$ & 0.0004 \\
\hline Moderate & $108(15.34)$ & $370(18.90)$ & 0.035 \\
\hline High & $22(16.30)$ & $55(15.63)$ & 0.86 \\
\hline All-cause (overall) & I,968 (6I.63) & $5,749(71.27)$ & $<0.0001$ \\
\hline \multicolumn{4}{|l|}{ COPD complexity } \\
\hline Low & $\mathrm{I}, 370(58.20)$ & $3,887(67.52)$ & $<0.000$ I \\
\hline Moderate & $496(70.45)$ & I,578 (80.59) & $<0.0001$ \\
\hline High & $102(75.56)$ & $284(80.68)$ & 0.21 \\
\hline
\end{tabular}

Notes: ${ }^{2}$ Those who had coexisting COPD and DM and received prescription for metformin during 2007-2010. 'Those who had coexisting COPD and DM and received antidiabetics prescription other than metformin, such as insulin, sulfonylurea, acarbose, exenatide, glucagon, miglitol, nateglinide, pioglitazone, pramlintide, repaglinide, rosiglitazone, saxagliptin, or sitagliptin during 2007-2010. ' $\chi^{2}$ test. Abbreviations: COPD, chronic obstructive pulmonary disease; DM, diabetes mellitus; ER, emergency room.

is more amenable to modification in the earlier stages of the disease. In advanced COPD, metformin may not be able to reduce excessive spillover of pulmonary inflammation, or merely reducing inflammation may not be enough to abate pulmonary symptoms. This inflammatory effect likely explains the benefit of metformin in patients with low COPD complexity.

Congestive heart failure (CHF) and chronic kidney disease (CKD) are common in patients with COPD and diabetes. ${ }^{33}$ All-cause ER visits and hospitalizations were decreased in both low-complexity and moderate-/high-complexity patients and consistent with the recent findings of a systematic review by Crowley et al that CHF and CKD patients on metformin had fewer CHF readmissions and lower allcause mortality. ${ }^{34}$ The role of metformin in the exacerbation of COPD without diabetes was studied by Hitchings et al. After metformin was given to patients during an acute exacerbation of COPD for 4 weeks, there was no significant difference in inflammatory markers as $\mathrm{C}$-reactive protein (CRP) or in clinical parameters measured by the COPD Assessment Test score and the EXAcerbations of Chronic Pulmonary Disease Tool score. ${ }^{35}$ The null effects were likely due to the short duration of therapy. Sexton et al found that 6 months of metformin improved respiratory symptoms and respiratory muscle strength and resulted in a trend toward a decrease in CRP. ${ }^{16}$ Cameron et al found that inflammatory markers were below the baseline after $8-12$ months of metformin use. ${ }^{11}$ Apparently, a longer duration of therapy is needed to show the beneficial effect of metformin.

\section{Limitations}

Our study findings should be interpreted within the context of the following limitations. First, our study is limited to fee-for-service Medicare beneficiaries and may not be generalizable to the non-Medicare population. Second, we used ICD-9-CM codes to establish a diagnosis of COPD and DM; these codes do not reflect disease severity. Administrative database can only provide the percentage of patients with COPD who had a spirometry during the study period, but not the actual values to determine whether or not a patient has airway obstruction. However, we used an administrative algorithm to capture the COPD and DM severity based on prior publications. ${ }^{20,21}$ Third, systemic chronic diseases interact with each other. Whether the beneficial effects of metformin are due to better control of diabetes, in a less sick patient with fewer exacerbations, or the direct effects of metformin altering the natural course of COPD is uncertain and beyond the scope of our study. Fourth, the surrogate

Table 3 Odds of ER visits and hospitalizations in patients with coexisting COPD and DM after the initial antidiabetic prescription during 2007-2010

\begin{tabular}{|c|c|c|c|c|}
\hline \multirow[t]{4}{*}{ Antidiabetic use } & \multicolumn{4}{|c|}{ ER visits or hospitalizations odds ratio ( $95 \%$ confidence interval) ${ }^{a}$} \\
\hline & \multicolumn{2}{|l|}{ COPD-specific } & \multicolumn{2}{|l|}{ All-cause } \\
\hline & \multicolumn{2}{|c|}{ COPD complexity } & \multicolumn{2}{|c|}{ COPD complexity } \\
\hline & Low & Moderate/high & Low & Moderate/high \\
\hline \multicolumn{5}{|l|}{ Metformin use } \\
\hline Nob & Reference & Reference & Reference & Reference \\
\hline Yes $^{c}$ & $0.66(0.52-0.85)$ & $0.82(0.64-1.04)$ & $0.73(0.65-0.81)$ & $0.72(0.59-0.88)$ \\
\hline
\end{tabular}

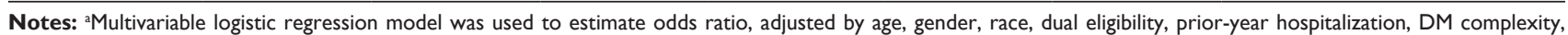
comorbidities (GERD, anxiety and depression, CVD, sarcopenia, and osteoporosis), prior-year oxygen use, and COPD maintenance medications use. 'bThose who had coexisting COPD and DM and received antidiabetics prescription other than metformin, such as insulin, sulfonylurea, acarbose, exenatide, glucagon, miglitol, nateglinide, pioglitazone, pramlintide, repaglinide, rosiglitazone, saxagliptin, or sitagliptin during 2007-20I0. 'Those who had coexisting COPD and DM and received prescription for metformin during 2007-2010.

Abbreviations: COPD, chronic obstructive pulmonary disease; CVD, cardiovascular disease; DM, diabetes mellitus; ER, emergency room; GERD, gastroesophageal reflux disease. 
Table 4 Odds of ER visits/hospitalizations in patients with coexisting COPD and DM after the index antidiabetic prescription during 2007-2010

\begin{tabular}{|c|c|c|c|c|}
\hline \multirow[t]{4}{*}{ Antidiabetics } & \multicolumn{4}{|c|}{ ER visits or hospitalizations odds ratio $\left(95 \%\right.$ confidence interval) ${ }^{\mathbf{a}}$} \\
\hline & \multicolumn{2}{|l|}{ COPD-specific } & \multicolumn{2}{|l|}{ All-cause } \\
\hline & \multicolumn{2}{|c|}{ COPD complexity } & \multicolumn{2}{|c|}{ COPD complexity } \\
\hline & Low & Moderate/high & Low & Moderate/high \\
\hline Insulin & Reference & Reference & Reference & Reference \\
\hline Metformin ${ }^{c}$ & $0.48(0.35-0.67)$ & $0.73(0.53-1.01)$ & $0.59(0.50-0.69)$ & $0.57(0.42-0.78)$ \\
\hline Sulfonylurea $^{d}$ & $0.60(0.44-0.83)$ & $0.81(0.59-1.11)$ & $0.69(0.58-0.81)$ & $0.68(0.50-0.93)$ \\
\hline Other ${ }^{e}$ & $0.70(0.53-0.93)$ & $0.94(0.7|-| .25)$ & $0.86(0.73-0.99)$ & $0.85(0.63-1.13)$ \\
\hline
\end{tabular}

Notes: aMultivariable logistic regression model was used to estimate odds ratio, adjusted by age, gender, race, dual eligibility, prior-year hospitalization, DM complexity, comorbidities (GERD, anxiety and depression, CVD, sarcopenia, and osteoporosis), prior-year oxygen use, and COPD maintenance medications use. ${ }^{\mathrm{T}}$ Those who had coexisting COPD and DM and received prescription for insulin during 2007-20I0. 'Those who had coexisting COPD and DM and received prescription for metformin during 2007-2010. 'Those who had coexisting COPD and DM and received prescription for sulfonylurea during 2007-2010. ${ }^{\mathrm{e}}$ Those who had coexisting COPD and DM and received antidiabetics prescription other than metformin, insulin, and sulfonylurea, such as acarbose, exenatide, glucagon, miglitol, nateglinide, pioglitazone, pramlintide, repaglinide, rosiglitazone, saxagliptin, or sitagliptin during 2007-2010.

Abbreviations: COPD, chronic obstructive pulmonary disease; CVD, cardiovascular disease; DM, diabetes mellitus; ER, emergency room; GERD, gastroesophageal reflux disease.

of medication use is prescription refill, and the use of this variable might not capture the actual use of and adherence to antidiabetics and COPD maintenance medication. Fifth, other cointerventions to prevent exacerbations such as smoking cessation, pulmonary rehabilitation, vaccination status, or the use of macrolides, roflumilast and mucolytic, were not examined. Sixth, the results of any observational study are subject to possible selection bias and unmeasured confounding. This is a hypothesis-generating study, and a randomized control trial should be performed to examine the effect of metformin in patients with COPD and DM.

\section{Conclusion}

Metformin use in Medicare patients with coexisting COPD and DM was associated with lower odds of COPD-specific ER visits and hospitalizations in patients with low-complexity COPD.

\section{Acknowledgments}

The authors thank Sarah Toombs-Smith, PhD, ELS, for help with the preparation of the manuscript. This work was supported by a grant (R01-HS020642) from the Agency for Healthcare Research. Funders had no role in the preparation, approval, or submission of the results. The abstract was presented in Annual CHEST Meeting, October 24, 2016, Los Angeles, CA (http://journal.chestnet.org/article/S0012$\underline{3692(16) 57195-5 / \mathrm{pdf}) \text {. }}$

\section{Disclosure}

Gulshan Sharma serves on the Advisory Board of Theravance Biopharma, Mylan, and Sunovion pharmaceutical companies. The other authors have no conflicts of interest in this work.

\section{References}

1. MacNee W. Is chronic obstructive pulmonary disease an accelerated aging disease? Ann Am Thorac Soc. 2016;13(Supplement_5):S429-S437.

2. Department of Noncommunicable Disease Prevention and Health Promotion, WHO. Active Ageing - A Policy Framework. Geneva: WHO; 2002:59.

3. Rogliani $\mathrm{P}$, Lucà G, Lauro D. Chronic obstructive pulmonary disease and diabetes. COPD Res Prac. 2015;1:3.

4. Faner R, Sobradillo P, Noguera A, et al. The inflammasome pathway in stable COPD and acute exacerbations. ERJ Open Res. 2016;2(3): 00002-2016.

5. Agustí A, Calverley PM, Decramer M, Stockley RA, Wedzicha JA. Prevention of exacerbations in chronic obstructive pulmonary disease: knowns and unknowns. Chronic Obstr Pulm Dis. 2014;1(2):166-184.

6. Wedzicha JA, Calverley PM, Rabe KF. Roflumilast: a review of its use in the treatment of COPD. Int J Chron Obstruct Pulmon Dis. 2016;11: $81-90$.

7. Rahman I. Pharmacological antioxidant strategies as therapeutic interventions for COPD. Biochim Biophys Acta. 2012;1822(5):714-728.

8. Poole P, Chong J, Cates CJ. Mucolytic agents versus placebo for chronic bronchitis or chronic obstructive pulmonary disease. Cochrane Database Syst Rev. 2015;(7):CD001287.

9. Pryor R, Cabreiro F. Repurposing metformin: an old drug with new tricks in its binding pockets. Biochem J. 2015;471(3):307-322.

10. Ito K, Colley T, Mercado N. Geroprotectors as a novel therapeutic strategy for COPD, an accelerating aging disease. Int J Chron Obstruct Pulmon Dis. 2012;7:641-652.

11. Cameron AR, Morrison VL, Levin D, et al. Anti-inflammatory effects of metformin irrespective of diabetes status. Circ Res. 2016;119(5): 652-665.

12. Hyun B, Shin S, Lee A, et al. Metformin down-regulates TNF- $\alpha$ secretion via suppression of scavenger receptors in macrophages. Immune Netw. 2013;13(4):123-132.

13. Calixto MC, Lintomen L, André DM, et al. Metformin attenuates the exacerbation of the allergic eosinophilic inflammation in high fat-dietinduced obesity in mice. PLoS One. 2013;8(10):e76786.

14. Park CS, Bang BR, Kwon HS, et al. Metformin reduces airway inflammation and remodeling via activation of AMP-activated protein kinase. Biochem Pharmacol. 2012;84(12):1660-1670.

15. Sato N, Takasaka N, Yoshida M, et al. Metformin attenuates lung fibrosis development via NOX4 suppression. Respir Res. 2016;17(1):107.

16. Sexton $P$, Metcalf $P$, Kolbe J. Respiratory effects of insulin sensitisation with metformin: a prospective observational study. COPD. 2014; 11(2):133-142. 
17. Hitchings AW, Archer JR, Srivastava SA, Baker EH. Safety of metformin in patients with chronic obstructive pulmonary disease and type 2 diabetes mellitus. COPD. 2015;12(2):126-131.

18. Research Data Assistance Center [homepage on the Internet]. CMS data file. Centers for medicare and medicaid services. Available from: https://www.resdac.org/cms-data. Accessed August 26, 2017.

19. Chronic Conditions Data Warehouse [homepage on the Internet]. Condition categories. Available from: https://www.ccwdata.org/web/ guest/condition-categories. Accessed August 26, 2017.

20. Mapel DW, Dutro MP, Marton JP, Woodruff K, Make B. Identifying and characterizing COPD patients in US managed care. A retrospective, cross-sectional analysis of administrative claims data. BMC Health Serv Res. 2011;11:43.

21. Kuo YF, Goodwin JS, Chen NW, Lwin KK, Baillargeon J, Raji MA. Diabetes mellitus care provided by nurse practitioners vs primary care physicians. J Am Geriatr Soc. 2015;63(10):1980-1988.

22. Li CY, Erickson SR, Wu CH. Metformin use and asthma outcomes among patients with concurrent asthma and diabetes. Respirology. 2016;21(7):1210-1218.

23. Papa EV, Dong X, Hassan M. Skeletal muscle function deficits in the elderly: current perspectives on resistance training. J Nat Sci. 2017;3(1): pii: e272.

24. Jones SE, Maddocks M, Kon SS, et al. Sarcopenia in COPD: prevalence, clinical correlates and response to pulmonary rehabilitation. Thorax. 2015;70(3):213-218.

25. Sreekumar R, Nair KS. Skeletal muscle mitochondrial dysfunction \& diabetes. Indian J Med Res. 2007;125(3):399-410.

26. Costa TM, Costa FM, Moreira CA, Rabelo LM, Boguszewski CL, Borba VZ. Sarcopenia in COPD: relationship with COPD severity and prognosis. J Bras Pneumol. 2015;41(5):415-421.
27. Jornayvaz FR, Shulman GI. Regulation of mitochondrial biogenesis. Essays Biochem. 2010;47:69-84.

28. Musi N, Hirshman MF, Nygren J, et al. Metformin increases AMPactivated protein kinase activity in skeletal muscle of subjects with type 2 diabetes. Diabetes. 2002;51(7):2074-2081.

29. Barceló B, Pons J, Fuster A, et al. Intracellular cytokine profile of T lymphocytes in patients with chronic obstructive pulmonary disease. Clin Exp Immunol. 2006;145(3):474-479.

30. Perret J, McDonald C, Apostolopoulos V. Elevated serum interleukin-5 levels in severe chronic obstructive pulmonary disease. Acta Biochim Biophys Sin (Shanghai). 2017;49(6):560-563.

31. Dal Negro RW, Bonadiman L, Turco P. Prevalence of different comorbidities in COPD patients by gender and GOLD stage. Multidiscip Respir Med. 2015;10(1):24.

32. Blasi F, Neri L, Centanni S, Falcone F, Di Maria G. Clinical characterization and treatment patterns for the frequent exacerbator phenotype in chronic obstructive pulmonary disease with severe or very severe airflow limitation. COPD. 2017;14(1):15-22.

33. Cavaillès A, Brinchault-Rabin G, Dixmier A, et al. Comorbidities of COPD. Eur Respir Rev. 2013;22(130):454-475.

34. Crowley MJ, Diamantidis CJ, McDuffie JR, et al. Clinical outcomes of metformin use in populations with chronic kidney disease, congestive heart failure, or chronic liver disease: a systematic review. Ann Intern Med. 2017;166(3):191-200.

35. Hitchings AW, Lai D, Jones PW, Baker EH; Metformin in COPD Trial Team. Metformin in severe exacerbations of chronic obstructive pulmonary disease: a randomised controlled trial. Thorax. 2016;71(7): 587-593.
International Journal of COPD

\section{Publish your work in this journal}

The International Journal of COPD is an international, peer-reviewed journal of therapeutics and pharmacology focusing on concise rapid reporting of clinical studies and reviews in COPD. Special focus is given to the pathophysiological processes underlying the disease, intervention programs, patient focused education, and self management protocols.

\section{Dovepress}

This journal is indexed on PubMed Central, MedLine and CAS. The manuscript management system is completely online and includes a very quick and fair peer-review system, which is all easy to use. Visit http://www.dovepress.com/testimonials.php to read real quotes from published authors. 